\title{
24. Tagung der Gesellschaft Junger Zivilrechtswissenschaftler
}

\author{
4.-7. September 2013, Bern, „Metamorphose des Zivilrechts“ \\ Johannes W. Flume*
}

Die diesjährige Tagung der "Jungen Zivilrechtswissenschaftler" fand in Bern statt. Assoziativ werden den meisten die, zurzeit drei, (Berner) Bären in den Sinn kommen, die arkadengesäumte mittelalterliche Altstadt, die Möglichkeit, sich in der gut temperierten und smaragdgrünen Aare treiben zu lassen und vielleicht auch ein Moment der Konfrontation mit der bekannten Berner Langsamkeit. Juristisch bot die auf der „großen Schanze“ mit Blick auf das Berner Oberland stattfindende Tagung den Teilnehmern den idealen Rahmen für das Aufeinandertreffen der drei deutschsprachigen Zivilrechtsnationen: Deutschland, Österreich und der Schweiz. Aus den unterschiedlichsten Gründen, sei es nun nationalistischer Zentrismus, Desinteresse oder schlicht fehlender Zugang, machen wir uns leider viel zu selten bewusst, wie gewinnbringend der komparatistische Blick zwischen diesen einzelnen Rechtsordnungen sein kann.

\section{Ein kurzer Appell für mehr komparatives (deutschsprachiges) Zivilrecht}

Bezogen auf den Austausch dieser drei Länder sollte man nicht vorschnell die Frage des Zusammenwirkens auf die Suche nach Verpflanzung von "legal transplants“ einschränken. Wie gewinnbringend der aktive Austausch zwischen den drei Ländern ist, wird schlagartig deutlich, wenn man zwei der berühmtesten Juristen der ersten Hälfte des 20. Jahrhunderts betrachtet, die den ständigen Vergleich der Rechtssysteme in ihrer Arbeit vorgelebt haben. Für das allgemeine Zivilrecht wird dies in den Werken von keinem Geringeren als Andreas von Tubr verkörpert. ${ }^{1}$ Wenn auch der Fokus seiner Werke maßgeblich von den unterschiedlichen Lebensphasen und -orten geprägt wurde, war die komparatistische Seitenschau stets gegenwärtig. ${ }^{2}$ Für das Gebiet des Handels- und Gesellschaftsrechts ist auf das zweibändige Werk ${ }^{3}$ von Karl Wieland zu verweisen. ${ }^{4}$ Für Wieland war die vergleichende Darstellung auch schon

* Dr. Johannes W. Flume ist wissenschaftlicher Mitarbeiter am Lehrstuhl für Bürgerliches Recht, Deutsche Rechtsgeschichte und Juristische Zeitgeschichte, Handels- und Gesellschaftsrecht (Prof. Dr. Jan Thiessen) an der Universität Tübingen.

1 Absolut lesenswert Andreas B. Schwarz, Andreas von Tuhr, Vortrag gehalten im Zürcherischen Juristenverein, Zürich 1938.

$2 \mathrm{Zu}$ verweisen ist dabei auf das in der Straßburger Zeit von Tuhrs entstandene Werk zum Allgemeinen Teil des BGB (Der Allgemeine Teil des Deutschen Bürgerlichen Rechts, Erster Band, Leipzig 1910, Zweiter Band, Erste Hälfte, Berlin 1914, Zweite Hälfte, Berlin 1918) sowie sein zweibändiges Werk zum Schweizer Obligationenrecht (Allgemeiner Teil des Schweizerischen Obligationenrechts, Erster Halbband, Tübingen 1924, Zweiter Halbband, Tübingen 1925); vgl. auch zu $v$. Tuhr zuletzt Hofer, in: Kölner Juristen im 20. Jahrhundert, Tübingen 2013, S. 5 ff.

3 Karl Wieland, Handelsrecht, Band I: Das kaufmännische Unternehmen und die Handelsgesellschaften, München 1921, Band II: Die Kapitalgesellschaften, München 1931.

$4 \mathrm{Zu}$ ihm und seinem Hauptwerk vgl. Karsten Schmidt, Morgenröte des Unternehmensrechts?, Ein Streifzug durch Wielands «Handelsrecht» von 1921 und 1931, in: Basler Juristische Mitteilungen (BJM), 2/2008, S. $61 \mathrm{ff}$. 
deshalb naheliegend, da die Entwicklung der handelsrechtlichen Kodifikationen zwischen Deutschland und Österreich von Beginn an auf das engste verknüpft und auch das zeitlich nachfolgende Schweizer Obligationenrecht im Diskurs mit den genannten handelsrechtlichen Kodifikationen entstanden war. ${ }^{5}$ Die Verwebungen sind zwischen Deutschland und Österreich besonders stark: Sie beginnen in der Mitte des 19 Jahrhunderts als, unter Zugrundelegung des preußischen Entwurfs eines Handelsgesetzbuchs von 1857 und unter Berücksichtigung der sog. österreichischen Entwür$\mathrm{fe}$, das ADHGB ausgearbeitet wurde. Während es, neben zahlreichen anderen deutschen Staaten, zuerst in Preußen 1861 eingeführt wurde, galt das ADHGB in Österreich ab 1863, unter Ausschluss des Seerechts, als AHGB. Im Jahre 1900 wurde das ADHGB im nun staatlich geformten Deutschland durch das HGB von 1897 ersetzt und neben das „neue“ BGB gestellt. In Österreich führt erst der „Anschluss“ Österreichs - auch dies ist ein Teil der Geschichte der Wechselwirkung zwischen den beiden Rechtsordnungen - von 1938 zur zwangsweisen Übernahme des deutschen Handelsrechts von 1897. Im kodifikatorischen Innovationswettkampf hat gegenwärtig Österreich durch die Umformung des HGB zu einem Unternehmensgesetzbuch (UGB) aus dem Jahre 2006 die Vorrangstellung übernommen. Eine ähnlich starke Verknüpfung lässt sich auch für das $\mathrm{GmbH}$ - und Aktienrecht nachweisen.

Mit diesem kleinen Abriss sollte exemplarisch demonstriert werden, wie stark die Querbezüge sind. Sie sind jedoch nicht nur auf die Gebiete des Handels- und Gesellschaftsrechts beschränkt, sondern lassen sich freilich auch für das bürgerliche Recht nachzeichnen. ${ }^{6}$ Nicht zuletzt aufgrund der fehlenden Sprachbarrieren kann der Austausch und die Berücksichtigung der deutschen, österreichischen und Schweizer Literatur ein ganz entscheidender Inspirationsgeber für die rechtswissenschaftliche Arbeit sein.

\section{Zum (General-)Thema}

Das Generalthema der Tagung lautete „Metamorphose des Zivilrechts“. Der Rektor der Universität Bern, Professor Martin Täubler, merkte in seinem Grußwort an, dass ihm als Naturwissenschaftler der Begriff des „,remodeling“ geläufiger sei. Am Beispiel des „bone remodeling“ beschrieb er jenen Prozess, der im Knochengewebe ständig stattfindet, um ein stabiles und funktionsfähiges System zu erhalten und es permanent an wechselnde Bedingungen anzupassen. Für die Wissenschaft ist dabei das Augenmerk auf den Umwandlungsprozess gerichtet, dessen Betrachtung und Analyse die Erkenntnis fördert. Als Gesellschaftsrechtler kommt einem unmittelbar der Wandlungsprozess von der Vor-Gesellschaft in eine juristische Person in den Sinn,

5 Zu den Wurzeln des Schweizer Handelsrechts Wieland, Handelsrecht, Bd. I. (Fn. 3), $\$ 6$ (= S. $36 \mathrm{ff}$ ).

6 Vgl. exemplarisch für das Schweizer ZGB Gmür, Das Schweizerische Zivilgesetzbuch, Verglichen mit dem deutschen Bürgerlichen Gesetzbuch, Bern 1965; siehe ferner auch die unterschiedlichen Beiträge zum 200- jährigen Bestehen des ABGB, Festschrift 200 Jahre ABGB, Band I. und II., Wien 2011. 
nämlich jener Prozess, der durch Eintragung in das Handelsregister abgeschlossen wird. ${ }^{7}$ Vielzitiert ist die Beschreibung von Erich Brodmann,${ }^{8}$, daß erst mit der Eintragung in das Handelsregister die Aktiengesellschaft da ist, nicht einen Augenblick früher, jetzt aber fertig und gerüstet [ist], wie Athene aus Jupiters Haupt“. Bei Wolfgang Schilling kann man dann nachlesen, dass Vorgesellschaft und Gesellschaft „ebenso identisch“ sind, „wie die Knospe und die Blüte, die Puppe und der Schmetterling, der Embryo und das Kind identisch sind “. ${ }^{9}$ Mit Rückgriffen auf die (Bilder) der Natur(-wissenschaften) muss man vorsichtig sein, da die Juristenzunft in einer Normordnung lebt, die sich nach der menschgeschaffenen Rechtsquellenlehre richtet. ${ }^{10}$ Wie dem auch sei, das Generalthema der Tagung gab einen interessanten Rahmen, der viele zivilrechtliche Fragestellungen beflügeln kann: Wie weit müssen wir die Grenzen und Bedingungen eines Rechtsinstituts verändern, so dass das ursprüngliche Institut X die Funktion Y übernimmt? Welches sind die möglichen Faktoren, die einen solchen Prozess einleiten können? Wie hat sich diese Entwicklung in der sog. horizontalen und vertikalen Rechtsvergleichung entwickelt?

\section{Die Referate}

Die Referate der Tagung waren in vier Blöcke eingeteilt. Den Vortragsblock am Donnerstag eröffnete Anne-Christine Mittwoch (Bremen) mit einem Beitrag zu den Harmonisierungskonzepten der europäischen Privatrechtsvereinheitlichung. Sie legte die Unterschiede zwischen der Mindest- und Vollharmonierung dar und sprach sich positiv zugunsten optionaler Regelungswerke aus. Julia Told (Wien) präsentierte einen engagierten und illustrativen Vortrag zur Wandlung des Verständnisses der Rechtsfähigkeit der Gesellschaft bürgerlichen Rechts. Der rechtsvergleichende Vortrag war ganz im Sinne des oben beschriebenen komparatistischen Ansatzes. Für das österreichische Recht liegt aufgrund der Normstruktur, des ABGB-Textes von 1811 und des UGB die Verneinung der Rechtsfähigkeit nahe; ein Reformvorhaben ist erst vor Kurzem im Sande verlaufen. Kontrovers aufgenommen wurde die These, dass die Rechtsfähigkeit der GbR und mit ihr auch die Gruppen-Lehre Werner Flumes allein auf einem Argument der „Natur der Sache“ beruhen solle, eine Argumentationsfigur, die man allenfalls mit Otto von Gierke verbunden hätte. Empirische Befunde zur Rechtsformwahl von Rechtsanwälten in Österreich wurden im Anschluss von Elisabeth Reiner (Wien) vorgestellt. Den Vortragsblock beschloss Barbara Łysz-

7 Hierzu zuletzt umfassend aus der Sicht des österreichischen Rechts U. Torggler, Die Verbandsgründung - de lege lata betrachtet, Wien 2009.

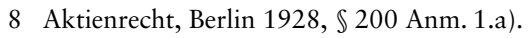

9 Schilling, in: Hachenburg, GmbHG, 6. Aufl., Berlin 1956, $\mathbb{1 1}$ Anm. 1; siehe hierzu auch Karsten Schmidt, Zur Stellung der OHG im System der Handelsgesellschaften, Bonn 1972, S. 310: „mehr geschadet als genützt".

10 Siehe in diesem Zusammenhang die Ausführungen von Theodor Kipp, Über Doppelwirkungen im Recht, insbesondere über die Konkurrenz von Nichtigkeit und Anfechtbarkeit, in: FS Ferdinand von Martitz, Berlin 1911, S. 211 ff. 
czarz (Wien) mit einem Referat zum Regelungskonzept der Dienstleistungsverträge des DCFR.

Der zweite Vortragstag wurde eingeleitet von Alexander Stöhr (Marburg) mit einer Zusammenstellung von Rechtsfragen, die das Internet im Zivilrecht provoziert hat. Dem folgten drei prägnante rechtshistorische und teils rechtsvergleichende Vorträge. Benjamin Bukor (Wien) ging auf das Abstammungsrecht des ABGB ein und inwieweit die nationalsozialistische Ideologie als treibende Kraft der Rechtsentwicklung und Rechtsprechung zum Zuge kam. Ihm folgte Michael Zwanzger (Bayreuth), der ein fulminantes geschichtliches Panorama zeichnete vom ideologischen Aufbau und der normativen Umsetzung des Reichserbhofgesetzes durch die Nationalsozialisten und wie dieses Modell letztlich kläglich scheiterte aufgrund der Widerstände in der bäuerlichen Gesellschaft, aber auch der wirtschaftlichen Defizite des Modells. Wolfram Buchwitz (Bonn) betrachtete in einem kurzen Abriss die rechtsdogmatische Entwicklung des Instituts der Ersitzung und stellte in einer rechtsvergleichenden Übersicht mögliche Rechtsinstitute vor, die sachenrechtlich, wenn sie nur entsprechend modifiziert werden, dieselbe Funktion wie die Ersitzung gleichsam übernehmen können. Der leider einzige Schweizer Beitrag von Daniel M. Häusermann (St. Gallen) präsentierte ein wahrlich Schweizer Thema: Inwieweit nämlich die seit 1891 bestehende Möglichkeit der Volksinitiative als „Motor der Zivilrechtsreform“ gewirkt hat. Überwiegend war dies im Gebiet des Sozial- und Arbeitsrechts der Fall. Internationale Bekanntheit hat aber auch die jüngst gesetzlich umgesetzte „Abzockerinitiative“ erlangt, in der die Begrenzung der Gehälter von Vorständen in Aktiengesellschaften festgelegt wurde. Dem folgte ein Vortrag von Wojciech Plesińki (Bayreuth) zum Aufeinandertreffen öffentlich-rechtlicher und zivilrechtlicher Regelungsmassen im Recht der Bauträgerverträge.

Das Interesse des letzten Tags galt dem Verbraucherrecht. Elke Heinrichs (Graz) Referat war der Bonitätsprüfung der Banken gewidmet; ein besonderes gesetzliches Regelungswerk musste von den nationalen Gesetzgebern jüngst aufgrund der Verbraucherkreditrichtline umgesetzt werden. Es zeigte sich im Vortrag wie auch in der nachfolgenden Diskussion, wie schwierig es einmal mehr sein kann, das Regelungsziel des europäischen Gesetzgebers zu erfassen und mit den nationalen Zivilrechtssystemen und Sanktionen in Einklang zu bringen. Dem folgte ein Vortrag von Philipp Fidler (Wien), der im Rahmen eines deutsch-österreichischen Vergleichs ein Paradethema des rechtsgeschäftlichen Massenverkehrs behandelte: Die Kontrolle von Allgemeinen Geschäftsbedingungen. Den Abschluss machte Simon Laimer (Innsbruck) mit einem kaufrechtlichen Referat, das die paternalistischen Einengung der Privatautonomie in diesem Gebiet zur Sprache brachte. 


\section{Das Besondere des Zusammentreffens der „Jungen“}

Regula Kurzbein, die Vorsitzende der GJZ und Mitveranstalterin der Berner Tagung, brachte bei der Eröffnung die Hoffnung zum Ausdruck, dass sich die Möglichkeit zum (Wieder-)Treffen und intensiven Austausch biete. Das hatte sich für mich persönlich von der ersten Minute an erfüllt. Ich hatte einen Kollegen und alten Studienfreund aus meiner Regensburger Zeit getroffen. Wir hatten uns aus den Augen verloren und waren uns nun in Bern zufällig begegnet. Diese persönliche Note in meinem Tagungsbericht mag eine wichtige Essenz der Treffen der "Jungen“ zum Ausdruck bringen. Fern von der Heimatfakultät können sich die wissenschaftlichen Mitarbeiter, Doktoranden und Habilitanden treffen, um zu erfahren, über welche Themen geforscht wird, wo und von wem. Man kann dabei auch in Erfahrung bringen, wie ganz unterschiedliche Themen angedacht werden und wer da sonst noch in den Republiken an der „academia“ Interesse hat. Das Berner Veranstaltungsteam hatte einen formidabel gastfreundlichen Empfang bereitet. Das Rahmenprogramm führte zu ein paar der schönsten Orte in und um Bern, an den letzten - wie es derzeitig scheint - sommerlichen Tagen dieses Jahres.

Die kommende Tagung wird vom 10.-13.9.2014 in Köln zum Thema „Richterliche Rechtsfortbildung und Kodifikation von Richterrecht" stattfinden. ${ }^{11} 2015$ werden sich die „Jungen“ in Marburg zusammenfinden. Man darf gespannt sein.

11 Zur Einstimmung vgl. Dauner-Lieb, Kodifikation von Richterrecht, in: Ernst/Zimmermann (Hrsg.), Zivilrechtswissenschaft und Schuldrechtsreform, Tübingen 2004, S. 305 ff. 